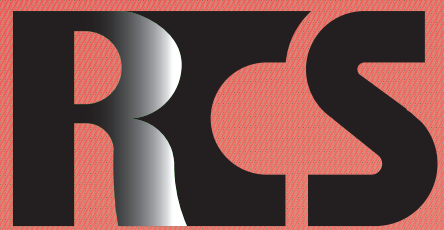

Depósito legal ppi $201502 Z U 4662$

Esta publicación científica en formato digital es continuidad de la revista impresa Depósito Legal: pp $197402 Z U 789$

- ISSN: 1315-9518 • ISSN-E: 2477-9431

Revista de Ciencias Sociales

Universidad del Zulia. Revista de la Facultad de Ciencias Económicas y Sociales Vol. XXVII. No. 2

Abril-Junio 2021

Esta publicación científica en formato digital es continuidad de la revista impresa Depósito Legal: pp $197402 Z$ Z789 ISSN: 1315-9518 

Ospino, C. (2021). Determinantes del desempeño gerencial: Instituciones de Educación Superior del departamento Atlántico-Colombia frente al Covid-19. Revista de Ciencias Sociales (Ve), XXVII(2), 296-308.

\title{
Determinantes del desempeño gerencial: Instituciones de Educación Superior del departamento Atlántico-Colombia frente al Covid-19
}

\author{
Acosta, Felipe* \\ Viloria, Daniel"* \\ Arteta, Ana *** \\ Ospino, César ${ }^{* * * *}$
}

\section{Resumen}

El objetivo de esta investigación fue caracterizar los elementos determinantes del desempeño gerencial en Instituciones de Educación Superior del departamento del Atlántico-Colombia frente al COVID-19. Metodológicamente, es una investigacion descriptiva con un diseño no experimental, transeccional y de campo. La población estuvo integrada por (54) docentes constituyentes de 4 instituciones de gestión pública y privada del departamento del Atlántico-Colombia. Como técnica de recolección de datos se usó la observación mediante encuesta y como instrumento la entrevista. La validez se realizó a través del juicio de expertos y la confiabilidad se determinó mediante el coeficiente Alfa de Cronbach alcanzándose los valores 0,91. El análisis de los datos fue mediante estadística descriptiva, estimándose medidas de tendencia central, así como de dispersión. Los resultados indican que en las instituciones de educacion superior estudiadas, se ha generado un proceso de integración del personal académico y administrativo, promoviendo un ambiente de trabajo idóneo, una vision de modernización de los procesos, la autoregulación del docente para impartir las cátedras, asi como una filosofía de vida que permite responder a los procesos socio familiares que ha traído el COVID 19. En conclusión, se evidenció un desempeño gerencial caracterizado por cumplimiento de aspectos propios de la labor directiva.

Palabras clave: Desempeño gerencial; responsabilidad; estrategias; Covid-19; instituciones de educación superior.

Doctor en Ciencias Económicas. Docente de la Fundación Universitaria de Popayán, Colombia. E-mail: felipe. acosta@fup.edu.co iD ORCID: https://orcid.org/0000-0001-6439-617X

** Magister en Ciencias de la Comunicación. Docente de la Universidad del Atlántico, Colombia. E-mail: danielviloria@, mail.uniatlantico.edu.co iD ORCID: https://orcid.org/0000-0002-2400-9128

*** Magister en Contabilidad Internacional y de Gestión. Docente de la Universidad de la Costa, Colombia. E-mail: aarteta4@cuc.edu.co iD ORCID: https://orcid.org/0000-0002-7151-2201

Maestría en Administración de Empresas e Innovación. Docente de la Universidad de la Costa, Colombia. E-mail: cospino1@cuc.edu.co iD ORCID: https://orcid.org/0000-0002-1399-5460 


\title{
Determinants of managerial performance: Higher Education Institutions of the Atlántico-Colombia department against Covid-19
}

\begin{abstract}
The objective of this research was to characterize the determining elements of managerial performance in Higher Education Institutions of the department of Atlántico-Colombia against COVID-19. Methodologically, it is a descriptive research with a non-experimental, transectional and field design. The population was made up of (54) constituent teachers from 4 public and private management institutions of the department of Atlántico-Colombia. As a data collection technique, observation through a survey was used and the interview as an instrument. Validity was carried out through expert judgment and reliability was determined using Cronbach's alpha coefficient, reaching values of 0.91 . The data analysis was by descriptive statistics, estimating measures of central tendency, as well as dispersion. The results indicate that in the higher education institutions studied, a process of integration of academic and administrative staff has been generated, promoting an ideal work environment, a vision of modernization of the processes, the self-regulation of the teacher to teach the chairs, as well as a philosophy of life that allows responding to the socio-family processes that COVID 19 has brought. In conclusion, a managerial performance characterized by compliance with aspects of directive work was evidenced.
\end{abstract}

Keywords: Managerial performance; responsibility; strategies; Covid-19; higher education institutions.

\section{Introducción}

Durante el año 2020 hubo una expansión de un virus a nivel mundial, cuyo origen fue encubado en China, el mismo denominado COVID-19, y fue tomando mucha fuerza al tiempo que iban evolucionando los meses, a tal punto que, para marzo de ese año, ya el virus se había expandido por todo el mundo. De acuerdo a lo planteado, los países fueron alcanzando posiciones y decisiones orientadas a resguardar a la población, estableciendo normativas, cuarentenas y límites para la supervivencia.

Martínez-Garcés y Garcés-Fuenmayor (2020) junto a Pérez, et al. (2020) y Macias (2020), coinciden en que, a pesar de las similitudes con pandemias anteriores, las características novedosas del COVID-19 señalan de manera clara la necesidad de investigar en relación con todos los factores de riesgo asociados a la vulnerabilidad ante la pandemia, que según Arana (2020), la situación vivida ha supuesto ya un aumento importante del nivel de estrés, que podría derivar en ansiedad a corto plazo y agravar problemas de salud y mentales previos o generar otros nuevos.

En función de poder responder a este problema de salud suscitado en Asia y que había transitado hasta América Latina, donde Colombia se había convertido en un alto receptor del COVID-19, el Gobierno Nacional estableció unos procedimientos para poder responder a esta situación critica y que permitiera evolucionar en algunas áreas, entre ellas la educacion. Para ello, las instituciones universitarias asumieron la responsabilidad de seguir funcionando a través del teletrabajo organizando y sustentado las plataformas tecnológicas con las que contaba y que eran utilizadas en forma irregular o como apoyo a 
los procesos.

Ante esta situación, surgió la intencionalidad de producir una nueva cultura universitaria, sustentada en diferentes practicas docentes orientadas a la gestion gerencial con base tecnológica, donde el teletrabajo se fue asumiendo como la base del proceso educativo, cuyas acciones están encauzadas a estimular el desarrollo de las capacidades cognitivas del docente a través del fortalecimiento de sus habilidades, lo cual trascendió de la vision académica, manifestándose el desarrollo biopsico-social del individuo, tomando en cuenta el desarrollo creativo, dado que el docente estaba condicionado a sus aulas de clase y solo hacía uso de las plataformas para respaldos o revisión esporádica (Duran, Sierra y Castro, 2019; Martínez-Garcés, Burbano-Vallejo y Burbano-Vallejo, 2019).

En virtud de lo expuesto, para hacer frente al COVID-19, en las Instituciones de Educación Superior (IES) colombianas se configuró la responsabilidad de adaptar y moldear una sociedad de conocimiento, para lograr dar respuesta a sus stakholders (docentes, estudiantes, empleados administrativos), dado que al cerrar las universidades se corría el riesgo de un alto nivel de desempleo, como ya se estaba dando en otras áreas de la economía mundial.

Al lograr lo anterior, se convertirían en organizaciones consolidadas con fuertes estructura académico-científica, que demostraría contar con capacidad técnica gerencial, además destacando con el respaldo de un capital humano, científico y tecnológico, situado en los más altos estándares de eficiencia para gestionar una academia con calidad, enmarcada en una concepción basada en una respuesta emergente ante la crisis mundial y que los estudiantes pudieran ir avanzando con clases virtuales (Barbera, Hernández y Vega, 2020; Díaz y Serra, 2020; Garcés y Mora, 2020).

Dentro de este marco, según García, et al. (2018) se sustenta que las organizaciones para cumplir sus metas en la sociedad, deben prestar servicios y productos de calidad, siendo eficientes como productivas, dedicarse a deleitar a sus clientes, proveyéndolos de servicios y productos que satisfagan excediendo sus necesidades como sus expectativas. Indudablemente, que para alcanzar esta meta deben contar con estrategias que permitan responder a las necesidades que surja, tal y como ocurrió y prevalece hoy en día con el COVID-19, involucrando a cada uno de los empleados que conforman la organización.

En este contexto, es donde el desempeño gerencial asume un rol importante, concebido como un medio para que los directivos, con el apoyo del equipo, promuevan procesos y transformaciones tanto en las acciones a realizar como en la aplicación de estrategias coherentes con la situación que se ha generado, tomando en cuenta la filosofía de gestion administrativa y gerencial.

Atendiendo a estas consideraciones, la IES del departamento del Atlántico en Colombia, han asumido la responsabilidad de dar respuesta a los estudiantes, quienes no podían asistir de manera física a sus aulas de clase, pero consideraron las actividades virtuales, mostrándose como organizaciones de cambio social; para ello, estas universidades requieren contar con un sistema de gestión del desempeño que comprenda y contribuya al crecimiento de las personas promoviendo en ellas la autoregulación así como el autocontrol, en función de alcanzar mayores niveles de excelencia en su desempeño laboral, esto ha sido sustentado con apoyo psico social.

Las acciones que emprendieron las universidades, les ha permitido ser asertivos en el desarrollo de los procesos académicos y administrativos, además han tenido control sobre el manejo de la incertidumbre, equilibrio de los miedos hacia el fracaso, permitiendo consolidar el autoestima y las emociones de los docentes, en la búsqueda de resultados positivos en el quehacer académico, es decir, que contribuya a lo que se conoce como el desarrollo de las competencias básicas para el crecimiento laboral.

Se deduce que, para lograr las metas y responder a la situación del COVID-19, las IES han asumido diversos procesos de la administración de organizaciones, pero 
encauzados en un liderazgo efectivo, en este sentido, Parra, et al. (2019) consideran que este liderazgo es la estrategia que debe prevalecer en las organizaciones que se mueven en un mundo globalizado, dentro de ambientes cambiantes y altamente incierto, como se ha podido confirmar en este proceso de incertidumbre y crisis mundial que se ha expandido, con el virus.

También, se ha destacado según Castro, et al. (2020), la existencia de un proceso coherente de dirección respaldado por aspectos propios de la labor directiva, como son: Toma de decisiones colectivas, incentivos al personal y fomento del trabajo en equipo, tratando de generar cumplimento de metas, $\mathrm{y}$ un alto desempeño gerencial, que responda al medio. Para Koontz, Weihrich y Cannice (2017), en toda organización los gerentes están llamados a tener un rol preponderante en procesos de liderazgo, no sólo como agente de cambio, sino como actor principal que modela y estimula a sus subordinados a buscar la satisfacción de sus necesidades más altas cubriendo así con sus expectativas.

Como fácilmente puede apreciarse, el esfuerzo humano resulta vital para el funcionamiento de cualquier organización; si el elemento humano está dispuesto a proporcionar su esfuerzo, la organización marchará; en caso contrario, se detendrá. De aquí, que toda organización debe prestar primordial atención a su personal (recursos humanos). En tal sentido, para que las organizaciones, alcancen sus objetivos, deben trabajar bajo esquemas de interacción continua y cíclica con el medio ambiente, es decir adaptarse a los procesos de transformación y cambio generado por el entorno.

En este orden de ideas, en la complejidad de sus procesos las universidades cuentan con una estructura social muy diversa, en función de las plataformas tecnológicas con las que cuentan para responder ante las actividades de teletrabajo, capacitación al docente en los sistemas tecnológicos y de información entre otros. Dado esto, los investigadores se orientaron a caracterizar los elementos determinantes del desempeño gerencial en las
IES del Departamento del Atlántico-Colombia frente al COVID-19.

\section{Elementos del desempeño gerencial: Estrategia de supervivencia organizacional ante el COVID-19}

Quienes administran las organizaciones, tienen una gran responsabilidad en el cumplimiento de la gestión, dado que deben establecer los objetivos, luego crear o diseñar la estrategia, además de ejecutarla y tal vez lo más importante, evaluar el desempeño de quienes están a cargo de dicha estrategia, con el fin de determinar el buen o mal desenvolvimiento de estos en pro del éxito de su gestión gerencial.

Dentro de este marco, ser un buen gerente no es fácil; sin embargo, los buenos directores se hacen, no nacen. Es necesario comprender, que la gestión gerencial como proceso estratégico dentro de las organizaciones, debe sustentarse en las habilidades gerenciales de los gerentes, puesto que éstas deben encajar dentro de la particularidad de cada institución.

En opinión de Moya, López y Artigas (2018), las universidades han sido históricamente instituciones importantes para la comunidad, puesto que de ella obtienen beneficios gran cantidad de actores sociales. De tal modo, que estos recintos de estudios se dedican a los procesos de formación integral de sus educandos, para que crezcan dentro de su propio proyecto ético de vida; a su vez, fomentando la investigación científica así como tecnológica y promoviendo el desarrollo cultural e ideológico. De igual manera, las universidades deben relacionarse con un pensamiento innovador y de cambio continuo, para comprender no solo la práctica educativa al interior de la universidad sino reflexionar e investigar sobre ella. Entre los elementos del desempeño gerencial están los siguientes:

a. Cumplimiento de la misión. Todo plan o gestión estratégica debe contar con el establecimiento de una misión que responda a las necesidades de la institución, a fin de 
tener claro hacia donde se enfocará el esfuerzo basado en las oportunidades y fortalezas de la misma, así como también determinar hasta donde sé es capaz de trabajar, pues, con la misión se sabe cuáles son los limites donde se circunscribe esta.

En ese sentido, Robbins y Coulter (2014) recomiendan que una vez analizado el entorno, los gerentes deben "determinar y evaluar alternativas estratégicas y enseguida elegir las que aprovechan las fuerzas de la institución y explotan las oportunidades del ambiente, o bien corrigen las debilidades de la organización y menguan las amenazas" (p.185). Bajo este contexto, la misión identifica los propósitos y límites de la organización. Al respecto, David (2013) fortalece que la misión "es la declaración fundamental que le da el carácter constitutivo a la organización y a su acción" (p.87). La misión, determina la estructura de la organización, los criterios de asignación de recursos, las posibilidades de desarrollo de las personas.

Aunado a ello, la misión es un importante elemento de la planificación estratégica planteado por García, et al. (2017), donde determina que a partir de ésta es que se formulan objetivos detallados, que son los que guiarán a la empresa u organización. Por tanto, resulta imprescindible que los líderes y directivos en general, conozcan cuál es el concepto de misión, y mejor aún, cuáles son las diferentes concepciones que proponen diversos expertos en temas de negocios, para que tengan una visión más completa y aplicable del mismo.

b. Responsabilidad social. La Responsabilidad Social Empresarial (RSE), para Guédez (2008), es una combinación de aspectos legales, éticos, morales y ambientales; asimismo, es una decisión voluntaria, no impuesta, donde se establece como principal estandarte en su cultura organizacional, la ética, la moral, los principios cívicos y ciudadanos y todo lo referente a sus valores. En tal sentido, la responsabilidad social, no es una actitud filantrópica que arroja beneficios solo hacia el medio ambiente, los recursos naturales, sociales y humanos, sino que representa para la empresa una inversión que repercute en beneficios financieros y en la consolidación del desarrollo humano.

En el contexto de las universidades del departamento del Atlántico, es el resultado de la combinación entre los intereses de la empresa y las necesidades de la comunidad. Asimismo, la responsabilidad social está orientada a la satisfacción de necesidades internas y externas, basadas en un conjunto de niveles de responsabilidad por parte de una empresa, responsabilidades básicas, organizacionales y sociales; asimismo, al "compromiso con sus colaboradores, más allá del cumplimiento tácito de las responsabilidades contractuales o normativas demandadas por la ley" (RubioRodríguez, Estrada y Pedraza, 2020, p.181).

De acuerdo a esta perspectiva, Cortina y Conill (2014), asumen que la responsabilidad social de la empresa es una visión y filosofía empresarial, al tiempo que sostienen que ésta puede y debe jugar un rol más allá de generar utilidades o ganancias. Es también, una conciencia de lo que las empresas producen que tiene impactos directos e indirectos dentro y fuera de la compañía.

Es así entonces como las IES en estudio han asumido posiciones socialmente responsables, preocupándose por el bienestar de quienes trabajan en ella, tales como docentes, administrativos y estudiantes, demostrando solidaridad con su entorno clientes, proveedores y la comunidad, entre otros. Aunado a ello, De la Cuesta, De la Cruz y Rodríguez (2010), sostienen que, desde el punto de vista moral, las organizaciones y empresas económicas tienen un papel social que jugar en la sociedad, debido a que en estos momentos es de relevancia extraordinaria por el impacto social y medioambiental de sus actuaciones.

Destacando que impacta la ética individual de las personas que componen las organizaciones, pero esta ha traspasado hacia la organización como un todo, como un ente con responsabilidad propia; de allí, que el argumento a favor de la Responsabilidad Social, es pues un asunto moral que no entra a analizar los beneficios económicos 
que pudieran obtener las empresas. En este contexto, la idea es generar bienestar; en función de la remuneración, la comunicación interna; la vinculación con los proveedores; la relación entre los accionistas; y, el desempeño de los gerentes.

c. Determinación de objetivos. Los objetivos organizacionales, son herramientas que indican el curso de acción que la organización debe realizar para alcanzar la misión y visión diseñadas, bajo parámetros de administración estratégica de recursos humanos, monitoreo y control de gestión. Según Hernández (2011), los objetivos se definen como propósitos de la organización en función del tiempo, se refieren a un resultado concreto que se desea o se necesita lograr dentro de un periodo específico, ya sea a corto (menos de un año), mediano (entre uno y cinco años) o largo plazo (más de cinco años). Los objetivos sirven para el desarrollo de metas y definición de acciones.

De igual manera, según Robbins y Coulter (2014), los objetivos se podrán definir como los resultados específicos que pretende alcanzar una organización por medio del cumplimiento de su misión básica. Son esenciales para el éxito de la organización, porque establecen un curso de acción, ayudan a la evaluación, revelan prioridades, permiten la coordinación y sientan las bases para planificar, organizar, motivar y controlar con eficiencia. Debe además, tenerse presente que las estrategias son un medio para alcanzar los objetivos a largo plazo.

Por otra parte, Chiavenato(2011) enuncia que los objetivos son los resultados futuros que se espera obtener. Son las metas seleccionadas, que se pretenden alcanzar en cierto tiempo con determinados recursos disponibles o posibles. Así, los objetivos son pretensiones futuras que, una vez alcanzadas, dejan de ser objetivos para convertirse en realidad. Asimismo, Koontz, et al. (2012), manifiestan que los objetivos son los fines importantes a los que se dirigen las actividades organizacionales e individuales. Un objetivo, es verificable cuando al final del periodo es posible determinar si se cumplió o no. También, se dice que son parte de la formulación de la estrategia.

Dentro de estas perspectivas, el establecimiento de objetivos, ayuda a minimizar las dificultades que tienen muchos miembros de la administración y sus subordinados, para saber cuáles son sus objetivos actuales, identificándolos tanto para ello como para sus asociados, poniéndolos al día, y usándolos con eficacia en su trabajo administrativo.

d. Alineación de estrategias. El gerente de hoy debe sumergirse en las realidades del entorno, interactuar con sus elementos y crear una intención estratégica que no es cosa distinta que generar un punto de vista con respecto al futuro; aplicando sus estrategias gerenciales. Considerando el concepto de estrategias gerenciales, estas representan, según Francés (2006), los cursos de acción que una organización adopta a través de la actuación y conocimientos gerenciales, como medio para alcanzar sus metas y objetivos a largo plazo.

En este contexto de la IES, Tiramonti (2011), ha sustentado que existe la necesidad de asumir posiciones gerenciales y generar las estrategias, las cuales permitan administrar al personal y los recursos dispuestos para el proceso de aprendizaje. Por tanto, los actores dentro del ámbito educativo deben ser analíticos pues necesitan saber dividir un problema en sus componentes, revisarlos y luego llegar a una solución factible; para ser conceptual, deben ser capaces de ver la tarea entera y relacionarla con otras, actuando como mediadores, y asumiendo decisiones en el ámbito educacional.

De acuerdo con Castro (2010), en materia educativa, las estrategias gerenciales comprenden el conjunto de acciones, las cuales aluden a las actividades de planificación, organización, dirección y control educativo; a través de las cuales se pueden transformar factores como: Relaciones interpersonales, regulaciones técnicas, comunicación, toma de decisiones, entre otros conceptos, que promueven la actuación y desarrollo integral de los trabajadores de la organización; pues sirven para el diseño de planes, los cuales se 
transformarán en la consecución de objetivos o el cumplimiento de metas de la organización.

En lo que concierne a Porter (2009), las estrategias son las escogencias que hace una organización para alcanzar los objetivos que tiene propuestos para el mediano y largo plazo. En sus relaciones con el equipo de dirección, el directivo hace varias cosas: Informarse de situaciones y enfoques que puedan ayudarlo a tomar decisiones; presentar sus criterios y propiciar un intercambio; resolver situaciones de conflicto entre integrantes del equipo y entre diferentes unidades organizativas; y, finalmente, propiciar consenso y compromiso en la toma de decisiones.

Asimismo, Duran, Parra y García (2018), asumieron que las estrategias deben estar determinadas respectivas a tiempo, espacio y objetivos a cumplir; diseñadas bajo un plan. Por lo cual, es concebida como un proceso orientador, capaz de proporcionar lineamientos para cumplir objetivos, establecer recursos y determinar políticas. Lo que genera acciones de fortalecimiento en las IES del departamento del Atlántico-Colombia.

\section{Metodología}

La investigación se orientó siguiendo los criterios del estudio cuantitativo, al pretender caracterizar los elementos determinantes del desempeño gerencial en IES del departamento del Atlántico-Colombia frente al COVID-19. En este caso, la variable fue susceptible de medición numérica a través de una ecuación que describe las variaciones entre ellas. Dentro de este marco, se clasifica como descriptiva y relevante porque describe la variable en un momento dado.

Por otra parte, su diseño es no experimental, puesto que el estudio no involucró una manipulación deliberada de la variable, la misma fue observada en las IES del departamento del Atlantico, tal y como sucede en la realidad. En otro ámbito, es transaccional, porque los datos se recopilaron en un solo momento, lo que brinda una oportunidad para el desarrollo de aplicaciones de instrumentos.
Además, se dice que la investigación se realizó en sitio, porque los datos fueron recolectados directamente en el lugar de contacto con los sujetos del componente poblacional o del sitio de investigación.

Seguidamente, se contó con una población de 54 docentes de las universidades en estudio, considerando que esta población es finita por estar constituida por menos de cien mil unidades y es accesible, denominado censo poblacional. Para recolectar los datos se utilizó un instrumento tipo cuestionario con 12 preguntas cerradas, con cinco (5) posibilidades de respuestas tipo Likert. El cuestionario estuvo configurado por un conjunto de items, dirigidos a medir el nivel de información sobre el desempeño gerencial.

A este instrumento se le aplicó la validez de contenido, a través del juicio de experto, posteriormente, se procedió a determinar la confiabilidad a través del método Alfa Crombach, el cual dio como resultado un coeficiente 0,91. El análisis de los datos se hizo a través de la estadística descriptiva, los mismos fueron procesados a partir de un baremo de interpretación, este se construyó sobre la base de las puntuaciones utilizadas en la escala, la cual va de 1 a 5 . Ubicándose en los rangos muy bajos $(0-1,81)$, bajo $(1,82-2,60)$, medio $(2,61-3,40)$, alta $(3,41-4,20)$ y muy alta $(4,21-5)$.

\section{Resultados y discusión}

Para dar respuesta a la pregunta de investigación, se establecieron 12 interrogantes con diferentes alternativas de respuestas, destacando 4 elementos claves que permiten caracterizar el desempeño gerencial en las IES del departamento del Atlántico en Colombia.

La Tabla 1, muestra que el $91 \%$ de los docentes consideraron estar entre completamente de acuerdo y medianamente de acuerdo con el cumplimiento de la misión, solo el 9\% seleccionó la opción ni de acuerdo ni en desacuerdo. Destacando una media aritmética, mediana y moda en 4,0; considerándose un rango alto en sus resultados. Mientras que 
la dispersión es de 0,7 ; observándolo como muy alto respecto a los rangos estimados. De acuerdo a lo apreciado, en las Instituciones de Educación Superior, se ha considerado que para hacer frente al COVID-19 se establecieron acciones coherentes que facilitaran el cumplimiento de la misión de dichas instituciones.

\section{Tabla 1}

\section{Cumplimiento de la misión}

\begin{tabular}{|c|c|c|c|c|c|c|c|}
\hline \multicolumn{2}{|l|}{ Estadística } & \multirow{2}{*}{$\frac{\text { Nivel }}{\text { Muy alto }}$} & \multirow{2}{*}{$\begin{array}{c}\text { Baremo } \\
\geq 4,21 \leq 5,00\end{array}$} & \multirow{2}{*}{$\begin{array}{c}\text { Respuesta } \\
\text { Completamente de acuerdo }\end{array}$} & \multirow{2}{*}{$\begin{array}{l}\mathbf{F a} \\
39\end{array}$} & \multirow{2}{*}{$\begin{array}{l}\text { Prop } \\
0,72\end{array}$} & \multirow{2}{*}{$\begin{array}{l}\% \\
72\end{array}$} \\
\hline Media & 4,0 & & & & & & \\
\hline Mediana & 4,0 & Alto & $\geq 3,41 \leq 4,20$ & Medianamente de acuerdo & 10 & 0,19 & 19 \\
\hline Moda & 4,0 & Moderado & $\geq 2,61 \leq 3,40$ & Ni de acuerdo ni en desacuerdo & 05 & 0,09 & 09 \\
\hline \multirow[t]{3}{*}{ Desv. Estándar } & 0,7 & Bajo & $\geq 1,82 \leq 2,60$ & Medianamente en desacuerdo & 00 & 0,00 & 00 \\
\hline & & Muy bajo & $\geq 0,00 \leq 1,81$ & Completamente en desacuerdo & 00 & 0,00 & 00 \\
\hline & & & No. ítems 3 & No. sujetos 54 & 54 & & 100 \\
\hline
\end{tabular}

Fuente: Elaboración propia, 2020.

En este aspecto se ha considerado que, en el cumplimiento de las tareas, los docentes se involucraron con los objetivos de desarrollo y respuesta para los estudiantes, enfocando los esfuerzos en las fortalezas que involucran las habilidades y competencias docentes y la plataforma tecnológica con la que cuentan las instituciones que se analizaron.

Respecto a comprobar si las instituciones en estudios son socialmente responsables, se determinó que un $72 \%$ de los docentes entrevistados asumieron estar completamente de acuerdo y medianamente de acuerdo, tal como se aprecia en la Tabla 2, además de que un $19 \%$ optó por la alternativa ni de acuerdo ni en desacuerdo y un $9 \%$ por la opción medianamente en desacuerdo. Esto indica, que estas instituciones se perfilan en un proceso de asumir su responsabilidad social, destacando que el COVID-19, establecido mundialmente como un problema de salud pública, debe ser enfrentado por estas organizaciones para viabilizar los procesos educativos ofreciendo a sus integrantes bienestar y estabilidad, coincidiendo con los aportes de Araya-Castillo y Rivera-Arroyo (2021).

Tabla 2

Instituciones socialmente responsables

\begin{tabular}{|c|c|c|c|c|c|c|c|}
\hline Estadística & & Nivel & Baremo & Respuesta & $\mathbf{F a}$ & Prop & $\%$ \\
\hline $\begin{array}{l}\text { Media } \\
\text { aritmética }\end{array}$ & 4,4 & Muy alto & $\geq 4,21 \leq 5,00$ & Completamente de acuerdo & 28 & 0,52 & 52 \\
\hline Mediana & 4,3 & Alto & $\geq 3,41 \leq 4,20$ & Medianamente de acuerdo & 11 & 0,20 & 20 \\
\hline Moda & 5,0 & Moderado & $\geq 2,61 \leq 3,40$ & Ni de acuerdo ni en desacuerdo & 10 & 0,19 & 19 \\
\hline \multirow[t]{3}{*}{ Desv. Estándar } & 0,4 & Bajo & $\geq 1,82 \leq 2,60$ & Medianamente en desacuerdo & 05 & 0,09 & 09 \\
\hline & & Muy bajo & $\geq 1,00 \leq 1,81$ & Completamente en desacuerdo & 00 & 0,00 & 00 \\
\hline & & & No. ítems 3 & No. sujetos 54 & 54 & & 100 \\
\hline
\end{tabular}

Fuente: Elaboración propia, 2020. 
En este orden de ideas, las medidas estadísticas tales como la media aritmética fue de 4,4, mediana 4,3 y moda 5,0; determinando que el indicador se ubicó en el nivel muy alto. La dispersión de 0,4 indica que el indicador personas se mueve hacia el nivel alto. Caracterizando a estas instituciones, como aquellas que asumieron el rol de dar respuesta efectiva a la sociedad del departamento, donde los docentes pudieran conservar sus empleos, y los alumnos obtuvieran las atenciones requeridas sin cortar su plan de estudio, combinando los intereses de estas universidades, y las necesidades de la comunidad.

Otro elemento que se destaca en el desempeño gerencial en las IES del departamento del Atlántico en Colombia, es la determinación de los objetivos, en este contexto, en la Tabla 3 se muestra que el $100 \%$ de los docentes consideró que los líderes y directivos, organizaron acciones coherentes para cumplir con objetivos internos y externos, dado que las tendencias fueron completamente de acuerdo y medianamente de acuerdo.

Tabla 3

Determinación de objetivos

\begin{tabular}{llllllll}
\hline Estadística & & Nivel & Baremo & Respuesta & Fa & Prop & $\%$ \\
\hline $\begin{array}{l}\text { Media } \\
\text { aritmética }\end{array}$ & 4,3 & Muy alto & $\geq 4,21 \leq 5,00$ & $\begin{array}{l}\text { Completamente de } \\
\text { acuerdo }\end{array}$ & 40 & 0,74 & 74 \\
Mediana & 4,4 & Alto & $\geq 3,41 \leq 4,20$ & $\begin{array}{l}\text { Medianamente de } \\
\text { acuerdo }\end{array}$ & 14 & 0,26 & 26 \\
Moda & 5,0 & Moderado & $\geq 2,61 \leq 3,40$ & $\begin{array}{l}\text { Ni de acuerdo ni en } \\
\text { desacuerdo }\end{array}$ & 00 & 0,00 & 00 \\
Desv. Estándar & 0,6 & Bajo & $\geq 1,82 \leq 2,60$ & $\begin{array}{l}\text { Medianamente en } \\
\text { desacuerdo }\end{array}$ & 00 & 0,00 & 00 \\
& Muy bajo & $\geq 1,00 \leq 1,81$ & $\begin{array}{l}\text { Completamente en } \\
\text { desacuerdo } \\
\text { No. sujetos 54 }\end{array}$ & $\mathbf{0 0}$ & 0,00 & 00 \\
& & No. ítems $\mathbf{3 4}$ & & $\mathbf{5 4}$ \\
\hline
\end{tabular}

Fuente: Elaboración propia, 2020.

En este ámbito, además se percibió una media aritmética de 4,3, la mediana de 4,4 y la moda de 5,0; lo que determina que el indicador se ubicó en el nivel muy alto, generando una dispersión de 0,6 lo que indica una alta dispersión respecto a los rangos de análisis. En este orden de ideas, las IES objeto de estudio, se orientaron a la determinación de objetivos concretos y específicos, que facilitara una respuesta a los integrantes de su comunidad, generando procesos que viabilizaran el momento de crisis de salud, pero que no cortara el desarrollo de las actividades académicas.

Es así como los objetivos se destacaron a corto plazo, y que pudieran ser medibles y cuantificables al término de cada semestre y se relacionaran con las disposiciones del
Gobierno Nacional, departamental y el municipal. Dando relevancia a preservar la salud y la vida de todos los miembros de su comunidad académica. Dentro de este marco, determinar los objetivos como matiz del desempeño gerencial donde se evidenciará el trabajo en equipo, se resaltan las necesidades, expectativas, intereses y comportamientos, de todas las partes.

En cuanto a la alineación de estrategias, se ha destacado este como un elemento clave del desempeño gerencial, y mucho más en tiempo de COVID-19, dado que los gerentes, directivos, lideres, entre otros, deben contrarrestar la situación de crisis país que se venía suscitando y debían enfrentar en forma coherentes. Es así como producto 
de la encuesta a docentes se obtuvo que el $93 \%$ de las tendencias consideraron estar completamente de acuerdo y medianamente de acuerdo, así también un 7\% que optó por la alternativa ni de acuerdo ni en desacuerdo, siendo neutral (ver Tabla 4).

Tabla 4

Alineación de estrategias

\begin{tabular}{|c|c|c|c|c|c|c|c|}
\hline Estadística & & Nivel & Baremo & Respuesta & $\mathbf{F a}$ & Prop & $\%$ \\
\hline Media aritmética & 4,0 & Muy alto & $\geq 4,21 \leq 5,00$ & Completamente de acuerdo & 37 & 0,69 & 69 \\
\hline Mediana & 4,0 & Alto & $\geq 3,41 \leq 4,20$ & Medianamente de acuerdo & 13 & 0,24 & 24 \\
\hline Moda & 4,0 & Moderado & $\geq 2,61 \leq 3,40$ & Ni de acuerdo ni en desacuerdo & 04 & 0,07 & 07 \\
\hline \multirow[t]{2}{*}{ Desv. Estándar } & 0,6 & Bajo & $\geq 1,82 \leq 2,60$ & Medianamente en desacuerdo & 00 & 0,00 & 00 \\
\hline & & Muy bajo & $\begin{array}{l}\geq 1,00 \leq 1,81 \\
\text { No. ítems } 3\end{array}$ & $\begin{array}{l}\text { Completamente en desacuerdo } \\
\text { No. sujetos } 54\end{array}$ & $\begin{array}{l}00 \\
\mathbf{5 4}\end{array}$ & 0,00 & $\begin{array}{l}00 \\
100\end{array}$ \\
\hline
\end{tabular}

Fuente: Elaboración propia, 2020.

Sobre las tendencias estadísticas de medida, la media aritmética, la mediana y la moda fueron de 4,0 en general, estableciendo una línea muy clara de los resultados, asumiendo una dispersión que fue de 0,6 respecto a los rangos establecidos en la desviación estándar. En este contexto, se observa que en las IES los líderes se orientaron a alinear las estrategias para consolidar procesos, que ya ellos tenían planteadas en otros ámbitos, las mismas se reestructuraron para hacer frente a la crisis de salud que está viviendo Colombia, las cantidades de casos que se estaban presentando y como afectaba a las familias el confinamiento. Concibiendo que los procesos de cuarentena afectan en los aspectos bio-psico y social al individuo, que en este caso los docentes debían promover procesos académicos que generaran en el estudiante involucramiento.

Además, de que los docentes tambien tenían confinamientos familiares y sociales que de diferentes maneras afecta el cumplimiento de objetivos. No obstante, la estrategia está orientada a responder a una situación de crisis de salud, pero también de escenarios emocionales familiares, que ha afectado de diferentes maneras a los miembros de la comunidad.

\section{Conclusiones}

De acuerdo a lo planteado en los puntos anteriores y más aún lo expresado en los resultados, el desempeño gerencial se relaciona con la competitividad, la cual puede ser vista como un tipo de relación influenciada por la disputa entre organizaciones, por recursos escasos, pero esenciales para sobrevivir. No obstante, dada la crisis de salud en el mundo, y la diversificación del COVID-19 en Colombia, las IES del departamento del Atlántico, asumieron con responsabilidad acciones coordinadas y determinadas a proteger los métodos que como organizaciones educativas tienen que cumplir en forma continua, pero que deben amparar en tiempo de crisis.

En este sentido, el COVID-19, como pandemia mundial ha afectado a un gran porcentaje de la población, generando altos niveles de estrés, de crisis emocional, problemas sociales; sin embargo, el estudio realizado ha demostrado que se coordinó desde las IES en forma coherente diferentes acciones, promoviendo un plan que en combinación con los entes del Estado se pudiera enfrentar este problema y tratar de apoyar a su comunidad con fortaleza.

Indudablemente, que para generar estas 
acciones se debía contar con un liderazgo efectivo, que estableciera un alto desempeño gerencial, que fuese capaz de alinear sus necesidades a una línea de respuesta a la sociedad y a sus miembros activos, donde los estudiantes pudieran recibir sus claves, apoyo emocional y psicológico y los docentes cumplieran su rol orientador así como el de facilitador de conocimiento.

Con el fin de consolidar el desempeño gerencial, se tomó como base el cumplimiento de la misión, ser una organización socialmente responsable, determinar objetivos acordes al problema que se está viviendo, asi como incluir las estrategias que estaban establecidas con las necesidades del entorno. El éxito o fracaso de estos procesos, y de supervivencia con el COVID-19, depende de la habilidad gerencial para lograr ventaja competitiva frente a las condiciones económicas, sociales, de emergencia en salud y de bienestar de la comunidad.

Esencialmente, se evidenció un desempeño gerencial caracterizado por cumplimiento de aspectos propios de la labor directiva, demostrándose toma de decisiones colectivas, iniciativas de responsabilidad social, establecimiento de objetivos, diseño de estrategias y fomento del trabajo en equipo. Bajo este contexto, en las IES se hizo necesario consolidar el compromiso de la alta dirección, formación del docente en plataformas tecnológicas, cambio de la mentalidad, asumiendo nuevos paradigmas, en este tiempo hubo una reducción de la resistencia a los cambios, y se promovieron innovaciones de los niveles de gestión intermedios.

\section{Referencias bibliográficas}

Arana, C. (2020). "El oriente de Cali no tiene ni Dios ni ley": Redes sociales digitales y violencia racializada a propósito de la COVID-19. SUMMA. Revista Disciplinaria en Ciencias Económicas y Sociales, 2(E), 217-243. https://doi. org/10.47666/summa.2.esp. 14
Araya-Castillo, L., y Rivera-Arroyo, J. (2021). ¿Cómo las instituciones de educación superior deben enfrentar los nuevos desafíos del entorno? Nota de actualidad. Revista de Ciencias Sociales (Ve), XXVII(1), 22-32. https:// doi.org/10.31876/rcs.v27i1.35293

Barbera, N., Hernández, E., y Vega, A. (2020). Desafíos de la gestión pedagógica en la virtualidad ante la crisis del COVID-19. SUMMA. Revista Disciplinaria en Ciencias Económicas $y$ Sociales, 2(E), 43-48. https://doi. org/10.47666/summa.2.esp.05

Castro, J. (2010). Nuevos aportes de la evaluación y calidad de la educación. Procesos y resultados. Cooperativa Editorial Magisterio.

Castro, B. R., Durán S. E., Mora, Y., Castro, R., Ballestas, M. D. R., y Tamara, J. (2020). El aprendizaje como eje estratégico del desarrollo organizacional en PYMEs de Barranquilla (Colombia). Revista Espacios, 41(41), 194-205.

Chiavenato, I. (2011). Administración de recursos humanos. El capital humano de las organizaciones. McGraw-Hill/ Interamericana Editores, S. A.

Cortina, A., y Conill, J. (2014). La responsabilidad ética de la sociedad civil. Mediterráneo Económico, (26), 13-29.

David, F. (2013). Strategic management: Concepts and cases. Pearson Education.

De La Cuesta, M., De La Cruz, C., y Rodríguez, J. M. (2010). Responsabilidad social universitaria. Netbiblo.

Díaz, A., y Serra, L. (2020). Competencias digitales del docente universitario. SUMMA. Revista Disciplinaria en Ciencias Económicas y Sociales, 2(1), 105-125.

Duran, S. E., Parra, M. A., y García, J. E. 
(2018). El aprendizaje como técnica de mejora continua en la gestión de desarrollo organizacional en Pymes Colombianas. El conocimiento como base del desarrollo económico y social entre los retos actuales, 1(1), 97-114.

Durán, S., Sierra, P., y Castro, R. (2019). El docente como agente promotor de la educación inclusiva y multicultural. En Universidad Pedagógica Experimental Libertador (Eds.), Memorias I Congreso Internacional de Investigación en Educación Superior (pp. 40-53). Fondo Editorial de la Universidad Pedagógica Experimental Libertador.

Francés, A. (2006). Estrategia y planes para la empresa: Con el cuadro de mando integral. Pearson Educación de México, S. A.

Garcés, J., y Mora, C. (2020). Estrategias de aprendizaje para mitigar la deserción estudiantil en el marco de la COVID-19. SUMMA. Revista Disciplinaria en Ciencias Económicas y Sociales, 2(E), 49-55.

García, J. E., Durán, S. E., Cardeño, E., Prieto, R., García, E., y Paz, A. (2017). Proceso de planificación estratégica: Etapas ejecutadas en pequeñas $\mathrm{y}$ medianas empresas para optimizar la competitividad. Revista Espacios, 38(52), 1-14.

García, J. E., Durán, S. E., Hernández, J. C., y Moreno, M. E. (2018). Estrategias gerenciales para fomentar las competencias laborales en el Sector Hotelero de la Costa Caribe colombiana. Revista de Investigación SIGMA, 5(2), 8-22.

Guédez, M. (2008). Responsabilidad social de las universidades hispanoamericanas para la animación de la cultura emprendedora regional. Universidad Nacional de San Martin.
Hernández, S. (2011). Introducción a la administración: Teoría general administrativa. Origen, evolución y vanguardia. McGraw-Hill.

Koontz, H., Weihrich, H., y Cannice, M. (2017). Elementos de la administración. McGraw-Hill.

Macías, A. J. (2020). Reacción de la sociedad civil y el tercer sector en el abordaje de las pandemias. Nota de actualidad. Revista de Ciencias Sociales (Ve), XXVI(2), 16-20. https://doi. org/10.31876/rcs.v26i2.32417

Martínez-Garcés, J., Burbano-Vallejo, M., y Burbano-Vallejo, E. (2019). Obstáculos y perspectivas al emplear tecnologías de información para enseñar contabilidad. Educación y Humanismo, 21(37), 104-119. https:// doi.org/10.17081/eduhum.21.37.3461

Martínez-Garcés, J., y Garcés-Fuenmayor, J. (2020). Competencias digitales docentes y el reto de la educación virtual derivado de la COVID-19. Educación y Humanismo, 22(39), 1-16. https:// doi.org/10.17081/eduhum.22.39.4114

Moya, F., López, D. D., y Artigas, W. (2018). Herramientas gerenciales desde el enfoque socio crítico en universidades: Estudio exploratorio en Venezuela y Colombia. Revista de Ciencias Sociales (Ve), XXIV(1), 101-112.

Parra, M., Visbal, O., Durán, S. E., y Badde, G. (2019). Calidad de la comunicación y actitud de los empleados ante procesos de cambio organizacional. Interdisciplinaria: Revista de Psicología y Ciencias Afines, (1), 155170.

Pérez, J. M., Dorado, A., Rodríguez, M. D. M., y López, J. (2020). Resiliencia para la promoción de la salud en la crisis COVID-19 en España. Revista de Ciencias Sociales (Ve), XXVI(4), 52-63. https://doi.org/10.31876/rcs. 
v26i4.34649

Porter, M. E. (2009). Ser competitivo. Ediciones Deusto.

Robbins, S. P., y Coulter, M. (2014). Administración. Editorial Pearson Education

Rubio-Rodríguez, G. A., Estrada, J. M., y Pedraza, G. (2020). Responsabilidad social universitaria: Incidencia en diferentes grupos de interés en una universidad colombiana. Revista de Ciencias Sociales (Ve), XXVI(4), 180189. https://doi.org/10.31876/rcs. v26i4.34656

Tiramonti, G. (2011). Educación secundaria Argentina: Dinámicas de selección y diferenciación. Cadernos de Pesquisa, 41(144), 692-709. https://doi.org/10.1590/S010015742011000300003 